\title{
Therapeutic Potential of Optogenetic Treatment for Individuals with Multiple Sclerosis
}

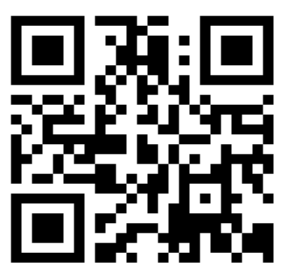

\author{
Mohamed M. El Koussy ${ }^{1 *} \&$ Nafisa M. Jadavji ${ }^{1}$
}

Multiple Sclerosis (MS) is a chronic neuroautoimmune condition characterized by neurodegeneration and demyelination throughout the central nervous system. While the pathology of MS is largely unknown, its symptoms are well defined. Current MS therapies such as intravenous corticoid injection, disease modifying treatments (DMTs) and neuro-rehabilitation exist; however most are ineffective as they do not manage symptoms efficeiently, leading to many adverse side effects. Optogenetic stimulation of demyelinated regions may serve as the needed therapy to effectively treat symptoms given the advances achieved in its rapid mechanisms and accurate cell-typespecific delivery strategies. In fact, the hallmark of optogenetic technology is the fast and accurate activation of specific neurons. Current evidence supports optogenetics as a means of controlling or enhancing neural circuitry involved in specific symptoms. This is done by targeting specific cells implicated in their respective neural circuits and activating them, or activating interneurons that inhibit the target pathway. Moreover, continuous photostimulation has been found to strengthen neuronal circuitry by promoting long-term potentiation (LTP). This review analyzes several studies that utilize optogenetics to alleviate MS-related symptoms such as cognitive impairment, visual impairment, bladder/bowel dysfunction, and tremors by controlling their specific pathways. It will also assess how these studies may translate to MS patients. Possible challenges in creating such a treatment will also be discussed. Given literature on the application of optogenetic treatment in neurodegenrative models is limited, this review presents a theoretical means of creating optogenetics treatment for MS and other neurodegenerative disorders.

\section{Introduction}

Multiple Sclerosis (MS) is a chronic autoimmune disease that leads to focal and diffuse neurodegenration and myelination throughout the nervous system (Kolasinski et al., 2012; Siffrin, Vogt, Radbruch, Nitsch, \& Zipp, 2010). In its most common form, relapseremitting MS, it is characterized by high inflammation levels that lead to a continuous cycle of relapse and remission (Raffel, Wakerley, \& Nicholas, 2016). These relapses, called exacerbations, may come in the form of new or worsening of old symptoms that are largely neurological such as visual impairment and imbalance that worsen over days or weeks, then recover spontaneously (Wingerchuk et al., 2014). Other common symptoms are cognitive impairment, loss of bladder control, leg weakness and sensory symptoms (Raffel et al., 2016).

Genetic and environmental factors both have a role in MS development; however, a specific link to the disease has not

${ }^{1}$ Department of Neuroscience, Carleton University, 1125 Colonel By Drive, Ottawa, ON K1S 5B6

*To whom correspondence should be addressed: moe.elkoussy@gmail.com

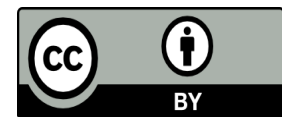

Except where otherwise noted, this work is licensed under https://creativecommons.org/licenses/by/4.0/

doi:10.22186/jyi.33.4.77-82 been found (Harbo, Gold, \& Tintoré, 2013). Genetically, MS is best characterized by a mutation on the human leukocyte antigen (HLA) gene locus, which causes abnormal antigen recognition of T cells leading to attacks on myelin proteins (Raffel et al., 2016). These findings have not been conclusive, as many other genes involved in immunological roles have also been found to play a role in contributing to MS. Environmental risk factors include smoking ,sunlight exposure, and vitamin D deficiency (Raffel et al., 2016).

Currently, there are no treatments that cure MS (Ziemssen et al., 2016). Instead, treatments target symptom management to increase patients' quality of life. These include high doses of corticosteroids such as methylprednisolone (Jongen et al., 2016), Disease modifying Treatments (DMTs) such as interferon $\beta-1 \mathrm{a}$, interferon $\beta-1 b$, alemtuzumab, fingolimod and natalizumab (Carrithers et al., 2014; Gajofatto \& Benedetti, 2015), and neuro-rehabilitation (Dasari, Wootla, Warrington, \& Rodriguez, 2016). All of these treatment options have adverse effects or are not particularly effective in the long term (Jongen et al., 2016; Ontaneda, Fox, \& Chataway, 2015; Schäcke, Döcke, \& Asadullah, 2002; Ziemssen et al., 2016). There is a considerable need for new treatment options that are more effective, while reducing the adverse side-effects. A potential therapy for MS-affected individuals may be the therapeutic application of optogenetics.

Optogenetics is a novel method that utilizes photoreceptors to selectively activate neurons (Hegemann \& Nagel, 2013). The genetic code of these receptors is delivered either virally or nonvirally to be expressed on the cells of interest. Once expressed, 
light is shone directly on these cells through an optic fiber inserted into the brain or spinal cord to be activated. Advances in this technology allow the photoreceptors to be selectively expressed on specific cell types, and in turn enable the control of specific neural pathways. Although largely used in research applications (Namboodiri \& Stuber, 2016), literature describing the therapeutic potential in neurodegenerative diseases is lacking. More recently, there has been a spike in literature demonstrating the therapeutic applications of optogenetics in the context of various disorders and symptoms. For example, evidence has shown optogenetic stimulation enhances cognitive function (Goshen, 2014), visual ability (Jazayeri \& Remington, 2016), bladder/bowel dysfunction (Stamp et al., 2017), and tremors (Tønnesen, 2013) through neuromodulatory effects.

While many of these studies have been conducted on specific symptoms, the focus of the optogenetic treatment has not been on any neurodegenerative models that express similar symptomology. Indeed, the current literature on the topic does not present any research on the translation of optogenetic treatment into any neurogenerative models expressing similar symptomology, requiring further research in this field (Ahmad, Ashraf, \& Komai, 2015; Bordia, Perez, Heiss, Zhang, \& Quik, 2016; Bryson, Machado, Lieberam, \& Greensmith, 2016; Jazayeri \& Remington, 2016). This review paper will assess the potential use of optogenetics in the development of therapies for MS related symptoms such as cognitive impairment, visual impairment, bladder/bowel dysfunction, and tremors.

\section{Development of Optogenetic Mechanisms}

Initial studies investigating the use of photoreceptors invovled metabotropic photoreceptors of vertebrate and invertebrate eyes (Zemelman, Lee, Ng, \& Miesenbö Ck, 2002). However, these systems were too complex to manipulate and the delay between light exposure and action potential firing was highly variable ranging from a few hundred milliseconds to tens of seconds (Lyon, 2013). Focus shifted to ionotropic microbial opsins as they exhibit fast, direct light-dependent ion conduction across the cell membrane (Mudiayi, Wong, \& Gruber, 2015). Furthermore, microbial opsins allow reversible control of neurons on the timescale of individual action potentials, which was lacking in earlier methods (Boyden Zhang, Bamberg, Nagel, \& Deisseroth, 2005). From a therapeutic perspective, however, the difference in seconds and milliseconds between the speed of activation of these cells is not significant. The rapid control of these cells does not necessarily affect the overall efficiency of the treatment. It is critical to understand that most optogenetic developments were directed as a means to enhance research tools. For example, creating faster optical controls allow remote control of individual spikes or synaptic events and enabling genetically targeted photostimulation with finer temporal resolution (Boyden et al., 2005). While the increased control speed is welcomed, it holds no major significance other than that symptoms would be halted seconds earlier. However, as it stands today, most studies use the microbial channelrhodopsin-2 (Nagel et al., 2003), warranting focus on this protein as a primary candidate for developing an optogenetic treatment.

ChR2 is isolated from the genome of the single celled algae Chlamydomonas reinhardtii (Nagel et al., 2003). ChR2 application was further developed by inserting the protein via viral vectors into mammalian hippocampal (HPC) neurons (Boyden et al., 2005). Once imbedded, high-speed optical switching photostimulates neurons, impressively responding in one to two milliseconds. Furthermore, neural activity was controlled by simply switching the optical blue light on or off (Boyden et al., 2005). This control can also be used by activating inhibitory circuitries, creating an antagonist effect on the region or function of interest. Molecularly, once the optical light is shone on the brain region of interest, photostimulation increased ion transport across the cellular membrane by either opening an ion channel or by actively pumping ions (Mudiayi et al., 2015).

\section{Current Delivery Strategies and Therapeutic Obstacles}

Non-viral delivery methods of expressing ChR2 in cells include in utero electroporation, transgenic models, chemical lipofection, and laser-assisted cellular poration (Boyden et al., 2005; Carter \& de Lecea, 2011; Mohanty \& Lakshminarayananan, 2015). Although beneficial for research, these methods are not viable translational strategies for human treatment. Moreover, these methods possess limitations such as not being able to specifically target cells, posing a risk to cellular components or the foreign DNA, and association with axonal pathology (Bryson et al., 2016; Mohanty \& Lakshminarayananan, 2015). Due to the lack in cell specific targeting and the lack of lateralization to humans of non-viral methods, it seems viral delivery methods provide the most sensible means of creating an optogenetic treatment, especially when studied in non-human primate models.

The most common method of expressing ChR2 in a nervous system is to infect neurons with a deficient virus replication, typically an adeno-associated virus (AAV) or lentivirus (LV), containing the transgene of interest driven by a short promoter or enhancer element (Carter \& de Lecea, 2011). AAVs are small viruses that efficiently transduce neurons while inducing minimal immune responses in the host brain (Blits et al., 2010). LV vectors are derived from a genus of retroviruses that cause chronic diseases characterized by long incubation periods such as the human immunodeficiency virus (HIV; Dull et al., 1998). In both methods, once ChR2 is expressed in the region of interest, illuminating the neurons with blue light at a bandwidth of 450-490 nm induces rapid depolarizing currents. However, literature has shown a difference in efficacy when used in non-human primates. One study has shown transduction with AAV yields positive functional and behavioral results, but not LV, indicating that AAV may be a more effective viral delivery method in primates compared to LV (Gerits et al., 2012). Moreover, in a recent breakthrough study, 
successful cell-type-specific expression of ChR2 in midbrain dopamine neurons of wild-type Rhesus macaques utilized AAVs, not LVs (Stauffer et al., 2016). A vector delivering Cre recombinase under the control of a tyrosine hydroxylase $(\mathrm{TH})$ promoter fragment and a vector delivering a Cre-recombinase-dependent ChR2 were mixed and injected to attain cell-type-specific expression of ChR2 (Stauffer et al., 2016). The TH promoter can be substituted in the first vector to other neuron-subtype-specific promoters to optogenetically control other neuron types in a monkey brain. For example, in an MS patient with lesions in the spinal cord affecting $\gamma$-aminobutyric acid (GABA) neurons, the Cre-recombinase vector being develived would require a glutamate decarboxylase (GAD) promoter fragment. This is because GAD is the enzyme that catalyzes the decarboxylation of glutamate to GABA and is only found in GABAergic cells, ensuring ChR2 expression is limited these cells. Further applications of this technique can be found in treatment of various MS symptoms utilizing the specific pathways and neural circuitry they operate through.

\section{Evidence of Therapeutic Potential for Multiple Sclerosis Re- lated Symptoms}

Photostimulation may serve as a factor in dealing with MS symptoms such as cognitive impairment (Goshen, 2014), visual impairment (Jazayeri \& Remington, 2016), bladder/bowel dysfunction (Stamp et al., 2017), and tremors (Tønnesen, 2013) by controlling their specific pathways. This especially possible once coupled with stem cell therapy (Bryson et al., 2016). However, while optogenetics may treat these symptoms, this review does not intend to demonstrate the effect of photostimulation on the autoimmune function of the disorder. To our knowledge, literature documenting immunomodulation using optogenetics is lacking, and what has been published only discusses proof-of-concept and designs for future development (Tan, He, Han, \& Zhou, 2017). The approaches involve optogenetic control of immune responses with novel tools that modulate lymphocyte trafficking, inflammasome activation, dendritic cell (DC) maturation, and antitumor immunity (Tan et al., 2017). Further information on the theoretical methods involving the combination of optogenetics and immunoengineering, termed optoimmunoengineering, can be found in the review conducted by Tan et al. (2017)

The most common cognitive impairment seen in MS is visual learning and memory (Chiaravalloti \& Deluca, 2008). Evidence has shown the problem lies in the initial learning of the memories as memory recall in MS patients is equal to healthy individuals, indicating that long-term memory systems are relatively intact (Chiaravalloti \& Deluca, 2008). The theoretical construct suggested to treat cognitive impairment in MS patients is to utilize induced pluripotent stem cells to derive oligodendrocyte progenitor cells and mature oligodendrocytes for remyelination of regions displaying degeneration that process working memory. While theroretically, stem cell transplantation should be able to resolve the issue with neuronal loss, clinical trials are showing unsuccessful results that are not entirely understood (Zhang et al., 2011). Recent studies suggest there is limited success due to the complexity involved with degrading glial scarring (Mallory, Grahn, Hachmann, Lujan, $\&$ Lee, 2015). It is hypothesized phototimulation of oligodendrocytes after differentiation would strengthen the remylination process as well as neural circuitry within that brain region through long-term potentiation (Lignani et al., 2013; Takeuchi et al., 2016). Takeuchi et al. (2016) were able to demonstrate optogenetic stimulation of the locus coeruleus (LC) enhances consolidation of everyday memory. The study electrophysiologically recorded LC firing rates in novel enivronments and stimuli and recreated this effect with photostimulation alone. Moreover, LTP was observed with repeated stimulation. This demonstration of LTP due to optogenetics is significant in providing contact for how optogenetics may affect neural circuitry with repeated stimulation. In this function and in the rest of the symptoms that will be discussed, we hypothesize that not only will optogenetics control the symptom by decreasing or inhibiting its presence, photostimulation may also lead to enhanced management of symptoms without the need for simulation in the long term.

Visual impairment is often seen in MS patients, commonly manifesting as optic neuritis, which is an acute inflammatory disorder of the optic nerve typically presenting with sudden monocular visual loss and eye pain (Garcia-Martin et al., 2017). Macaques were used to study visual information processing mechanisms in the lateral geniculate nucleus (LGN) and primary visual cortex (V1) by administering an AAV with an effective CamKII promoter into koniocellular cells (K-cells) at the LGN (Klein et al., 2016). The LGN is made up of K-cells, parvo cells and magno cells, each distinct in their circuitry, function and biochemistry, despite all passing through the V1. K-cells however were used as they are especially different form the other two cells. The vectors used were able to target K-cells, nearby CamKII-positive cells, as well as transduce distant layer 6 pyramidal cells of V1 and retinal ganglion cells (Klein et al., 2016). Measurements were conducted using average local field potential (LFP) responses across stimulation trials and current source-density (CSD) profiles were calculated for the visual flicker and optogenetic conditions to assess V1 laminar activation. Of the total population of LGN neurons recorded, the authors identified $23 \%$ as being directly affected by the optogenetic stimulation, in comparison to the $\sim 10 \%$ observed in the literature (Klein et al., 2016). Although less than $50 \%$ of the cells were activated, the authors were able to confirm that at the neuronal circuit level, the amount of selectively recruited K-cells was sufficient to drive short-latency activity in the supra-granular layers of downstream area V1 (Klein et al., 2016).

In translating these findings for an MS treatment, a more enhanced outcome may be observed if all cells of LGN are recuited. This is especially since this study only sought to assess the ability of optogenetic stimulation in the visual cortex to understand the visual pathway, not with the intention of developing a therapy for visual impairment. No mention was made on the level of enhancement observed in the visual ability of the monkeys, warranting further studies on optogenetic stimulation of this brain region. 
Moreover, an optogenetic treatement for this function coupled with stem cell therapy may provide a means to alter visual impairment in MS. Similar to the function described in treating cognitive impairment, photostimulation of these pathways may treat visual impairment by strengthening and expanding the neural circuitry and remyelination processes. Interestingly, clinical trials are being conducted for a fascinating treatment option for retinitis pigmentosa (RP) using ChR2 and the concept of optogenetic stimulation (Birch, 2016).

Bladder and bowel dysfunctions are commonly seen in MS cases, causing some of the most distressing symptoms with as many as $75 \%$ MS of patients presenting the symptom (Andretta, Simeone, Ostardo, Pastorello, \& Zuliani, 2014). The most frequent bladder symptoms seen are storage symptoms such as urinary frequency, urgency, and urge incontinence (Andretta et al., 2014). Voiding symptoms such as hesitancy, incomplete voiding and urinary retention are present as well, although to a lesser extent (Andretta et al., 2014).

Enteric neural cells from fetal or postnatal mouse bowels expressing ChR2 were transplanted into the distal colon of 3-4 week old wild-type mice (Stamp et al., 2017). The transplanted neural cells were able to differentiate into multiple functional types of neurons, integrating and providing functional innervation of the smooth muscle of the bowel wall (Stamp et al., 2017). In the study, optogenetics was used to to selectively stimulate graft-dervied neurons to identify that enteric neural cells isolated from the embryonic and postnatal bowels, giving rise to functional inhibitory motor neurons, excitatory motor neurons, and interneurons following transplantation into the distal colon of recipient mice (Stamp et al., 2017). While optogenetic stimulation was not the primary cause of the functional recovery, when used as a tool, photostimulation can control how these new cells operate. In a disorder that leads to a loss of function due to continuous degeneration, optogenetic stimulation can speed the recovery period as cells are continuously destroyed. Moreover, we hypothesize continuous photostimulation will lead to LTP and strengthening of these networks faster than without the stimulation. These findings are significant as they illuminate a fundamental limitation in almost all the current gold-standard nerve-targeted treatment approaches which do not specifically address isolated neural circuits and lead to undesirable side effects such as unwanted bowel movements or sexual function (Park et al., 2017).

Tremors are believed to occur in up to $75 \%$ of MS patients appearing in various forms such as postural, kinetic, proximal, distal tremors, and internal tremors (IT; Ayache et al., 2015). Dysfunction in inhibitory cerebellar efferent projections likely play a role in the generation of tremors during posture or movement in MS patients, however, additional lesions of other cerebral pathways might be involved (Ayache et al., 2015). Optogenetic treatment for tremors would function similarly to those documented in Parkinson's disease (PD). Cell-type specific targeting of dopaminergic neurons in the substantia nigra pars compacta $(\mathrm{SNc})$ has shown to be a viable means of treating denervation of striatal target areas
(Stauffer et al., 2016; Tønnesen, 2013). This process is thoroughly described in the study by Stauffer et al. (2016). Applying photostimulation to the regions found to underlie tremor development and motor dysfunction in MS, such as gait, would be a feasible means of treating these symptoms, given its documented applications in similar cases (Kravitz et al., 2010).

\section{Future Directions and Conclusion}

Optogenetics has been reported to be a revolutionary technique in neurobiology research. As such, the objective of this review is to assess the potential use of optogenetics in developing therapies for MS related symptoms that include cognitive impairment, visual impairment, bladder/bowel dysfunction, and tremors. Studies for each of these symptoms have been discussed and analyzed and two primary conclusions have been found. Intially, optogenetics remains a tool to enhance treatment. For example, in the study by Stamp et al. (2017), the primary function of the study was to understand how implanted enteric cells functionally integrate into endogenous cells. Optogenetics was merely a tool to control this function to further study it. While it was not used as a treatment, this function of controlling the circuitry may be manipulated into a therapy for those with bladder dysfunction and other illnesses observed in MS.

Secondly, while this control does require manually intiating photostimulation, continuous stimulation may lead to enhancement in circuitry through neuroplastic and LTP effects as seen in Takeuchi et al. (2016). We hypothesize the manual function of turning on photostimulation in the brain may work similarly to a pacemaker where optic fibers are connected internally in the body. Of course these concepts remain theoretical and require extensive research to validate this possibility.

Ultimately, furthering this therapeutic tool is limited by the knowledge present for specific symptoms as well as their mechanisms (Bryson et al., 2016; Jazayeri \& Remington, 2016; Mohanty \& Lakshminarayananan, 2015; Mudiayi et al., 2015). Just as optogenetics may be used as a therapeutic tool, it can be utilized to understand these symptoms as it is currently used as an investigative tool (Carter \& de Lecea, 2011; Mohanty \& Lakshminarayananan, 2015; Mudiayi et al., 2015). A first step to identifying how photostimulation can lead to therapeutic effects could ultimately be using optogenetics to understand individual symptoms by experimenting with its circuitry and inhibiting or activating symptom pathways. Once this knowledge has been attained, more specific and accurate targets can be found to control MS symptomology.

\section{REFERENCES}

Ahmad, A., Ashraf, S., \& Komai, S. (2015). Optogenetics applications for treating spinal cord injury. Asian Spine Journal, 9(2), 299-305. doi:10.4184/ asj.2015.9.2.299

Andretta, E., Simeone, C., Ostardo, E., Pastorello, M., \& Zuliani, C. (2014). Usefulness of sacral nerve modulation in a series of multiple sclerosis patients with bladder dysfunction. Journal of the Neurological Sciences, 347(1), 257 261. doi:10.1016/j.jns.2014.10.010

Ayache, S. S., Chalah, M. A., Al-Ani, T., Farhat, W. H., Zouari, H. G., Créange, A., \& Lefaucheur, J.-P. (2015). Tremor in multiple sclerosis: The intriguing role 
of the cerebellum. Journal of the Neurological Sciences, 358(1), 351-356. doi:10.1016/j.jns.2015.09.360

Birch, D. G. (2016). RST-001 Phase I/II Trial for Retinitis Pigmentosa. Retrieved February 28, 2017, from https://clinicaltrials.gov/ct2/show/NCT02556736?t erm $=$ optogenetics\&rank $=1$

Blits, B., Derks, S., Twisk, J., Ehlert, E., Prins, J., \& Verhaagen, J. (2010). Adenoassociated viral vector (AAV)-mediated gene transfer in the red nucleus of the adult rat brain: Comparative analysis of the transduction properties of seven AAV serotypes and lentiviral vectors. Journal of Neuroscience Methods, 185(2), 257-263. doi:10.1016/j.jneumeth.2009.10.009

Bordia, T., Perez, X. A., Heiss, J. E., Zhang, D., \& Quik, M. (2016). Optogenetic activation of striatal cholinergic interneurons regulates L-dopa-induced dyskinesias. Neurobiology of Disease, 91, 47-58. doi:10.1016/j.nbd.2016.02.019

Boyden, E. S., Zhang, F., Bamberg, E., Nagel, G., \& Deisseroth, K. (2005). Millisecond-timescale, genetically targeted optical control of neural activity. $\mathrm{Na}$ ture Neuroscience, 8(9), 1263-1268. doi:10.1038/nn1525

Bryson, J. B., Machado, C. B., Lieberam, I., \& Greensmith, L. (2016). Restoring motor function using optogenetics and neural engraftment. Current Opinion in Biotechnology, 40, 75-81. doi:10.1016/j.copbio.2016.02.016

Carrithers, M. D., Kobelt, G., Berg, J., Atherly, D., Hadjimichael, O., Scalfari, A., ... Radtke, C. (2014). Update on disease-modifying treatments for multiple sclerosis. Clinical Therapeutics, 36(12), 1938-45. doi:10.1016/j. clinthera.2014.08.006

Carter, M. E., \& de Lecea, L. (2011). Optogenetic investigation of neural circuits in vivo. Trends in Molecular Medicine, 17(4), 197-206. doi:10.1016/j.molmed.2010.12.005

Chiaravalloti, N. D., \& Deluca, J. (2008). Cognitive impairment in multiple sclerosis. The Lancet Neurology, 7, 1139-1151. doi:10.1016/S14744422(08)70259-X

Dasari, H., Wootla, B., Warrington, A. E., \& Rodriguez, M. (2016). Concomitant Use of Neuroprotective Drugs in Neuro Rehabilitation of Multiple Sclerosis. International Journal of Physical Medicine \& Rehabilitation, 4(4). doi:10.4172/2329-9096.1000348

Dull, T., Zufferey, R., Kelly, M., Mandel, R. J., Nguyen, M., Trono, D., \& Naldini, L. (1998). A third-generation lentivirus vector with a conditional packaging system. Journal of Virology, 72(11), 8463-71. Retrieved from http://www. ncbi.nlm.nih.gov/pubmed/9765382

Gajofatto, A., \& Benedetti, M. D. (2015). Treatment strategies for multiple sclerosis: When to start, when to change, when to stop? World Journal of Clinical Cases, 3(7), 545-55. doi:10.12998/wjcc.v3.i7.545

Garcia-Martin, E., Ara, J. R., Martin, J., Almarcegui, C., Dolz, I., Vilades, E., ... Satue, M. (2017). Retinal and Optic Nerve Degeneration in Patients with Multiple Sclerosis Followed up for 5 Years. Ophthalmology. doi:10.1016/j. ophtha.2017.01.005

Gerits, A., Farivar, R., Rosen, B. R., Wald, L. L., Boyden, E. S., \& Vanduffel, W. (2012). Optogenetically induced behavioral and functional network changes in primates. Current Biology : CB, 22(18), 1722-6. doi:10.1016/j. cub.2012.07.023

Goshen, I. (2014). The optogenetic revolution in memory research. Trends in Neurosciences, 37(9), 511-522. doi:10.1016/j.tins.2014.06.002

Harbo, H. F., Gold, R., \& Tintoré, M. (2013). Sex and gender issues in multiple sclerosis. Therapeutic Advances in Neurological Disorders, 6(4), 237-48. doi: $10.1177 / 1756285613488434$

Hegemann, P., \& Nagel, G. (2013). From channelrhodopsins to optogenetics. EMBO Molecular Medicine, 5(2), 173-176. doi:10.1002/emmm.201202387

Jazayeri, M., \& Remington, E. (2016). Optogenetics Advances in Primate Visual Pathway. Neuron, 90(1), 8-10. doi:10.1016/j.neuron.2016.03.024

Jongen, P. J., Stavrakaki, I., Voet, B., Hoogervorst, E., van Munster, E., Linssen, W. H., ... Gaillard, P. (2016). Patient-reported adverse effects of high-dose intravenous methylprednisolone treatment: a prospective web-based multicenter study in multiple sclerosis patients with a relapse. Journal of Neurology, 263(8), 1641-51. doi:10.1007/s00415-016-8183-3

Klein, C., Evrard, H. C., Shapcott, K. A., Haverkamp, S., Logothetis, N. K., \& Schmid, M. C. (2016). Cell-Targeted Optogenetics and Electrical Microstim- ulation Reveal the Primate Koniocellular Projection to Supra-granular Visual Cortex. Neuron, 90(1), 143-151. doi:10.1016/j.neuron.2016.02.036

Kolasinski, J., Stagg, C. J., Chance, S. A., Deluca, G. C., Esiri, M. M., Chang, E.H., ... Johansen-Berg, H. (2012). A combined post-mortem magnetic resonance imaging and quantitative histological study of multiple sclerosis pathology. Brain : A Journal of Neurology, 135(Pt 10), 2938-51. doi:10.1093/ brain/aws 242

Kravitz, A. V, Freeze, B. S., Parker, P. R. L., Kay, K., Thwin, M. T., Deisseroth, K., \& Kreitzer, A. C. (2010). Regulation of parkinsonian motor behaviours by optogenetic control of basal ganglia circuitry. Nature, 466(7306), 622-6. doi:10.1038/nature09159

Lignani, G., Ferrea, E., Difato, F., Amarù, J., Ferroni, E., Lugarà, E., ... Benfenati, F. (2013). Long-term optical stimulation of channelrhodopsin-expressing neurons to study network plasticity. Frontiers in Molecular Neuroscience, 6 , 22. doi:10.3389/fnmol.2013.00022

Lyon, L. (2013). Optogenetics. Materials and Methods, 3, 194. doi:10.13070/ mm.en.3.194

Mallory, G. W., Grahn, P. J., Hachmann, J. T., Lujan, J. L., \& Lee, K. H. (2015). Optical stimulation for restoration of motor function following spinal cord injury. Mayo Clin Proc, 90(2), 300-307. doi:10.1016/j.mayocp.2014.12.004

Mohanty, S. K., \& Lakshminarayananan, V. (2015). Optical Techniques in Optogenetics. Journal of Modern Optics, 62(12), 949-970. doi:10.1080/0950034 0.2015 .1010620

Mudiayi, D., Wong, S., \& Gruber, A. (2015). Optogenetics. International Encyclopedia of the Social \& Behavioral Sciences, 17, 268-273. doi:10.1016/B9780-08-097086-8.55060-0

Nagel, G., Szellas, T., Huhn, W., Kateriya, S., Adeishvili, N., Berthold, P., ... Bamberg, E. (2003). Channelrhodopsin-2, a directly light-gated cationselective membrane channel. Procedings of the National Academy of Sciences of the United States of America, 100(23), 13940-13945. doi:10.1073/ pnas. 1936192100

Namboodiri, V. M. K., \& Stuber, G. D. (2016). Cell-Type-Specific Optogenetics in Monkeys. Cell, 166(6), 1366-1368. doi:10.1016/j.cell.2016.08.047

Ontaneda, D., Fox, R. J., \& Chataway, J. (2015). Clinical trials in progressive multiple sclerosis: lessons learned and future perspectives. The Lancet. Neurology, 14(2), 208-23. doi:10.1016/S1474-4422(14)70264-9

Park, J. H., Hong, J. K., Jang, J. Y., An, J., Lee, K.-S., Kang, T. M., ... Suh, J.K. F. (2017). Optogenetic Modulation of Urinary Bladder Contraction for Lower Urinary Tract Dysfunction. Scientific Reports, 7, 40872. doi:10.1038/ srep 40872

Raffel, J., Wakerley, B., \& Nicholas, R. (2016). Multiple sclerosis. Medicine, 44(9), 537-541. doi:10.1016/j.mpmed.2016.06.005

Schäcke, H., Döcke, W.-D., \& Asadullah, K. (2002). Mechanisms involved in the side effects of glucocorticoids. Pharmacology \& Therapeutics, 96(1), 23-43. doi:10.1016/S0163-7258(02)00297-8

Siffrin, V., Vogt, J., Radbruch, H., Nitsch, R., \& Zipp, F. (2010). Multiple sclerosis - candidate mechanisms underlying CNS atrophy. Trends in Neurosciences, 33(4), 202-210. doi:10.1016/j.tins.2010.01.002

Stamp, L. A., Gwynne, R. M., Foong, J. P. P., Lomax, A. E., Hao, M. M., Kaplan, D. I., ... Young, H. M. (2017). Optogenetic demonstration of functional innervation of mouse colon by neurons derived from transplanted neural cells. Gastroenterology. doi:10.1053/j.gastro.2017.01.005

Stauffer, W. R., Lak, A., Yang, A., Borel, M., Paulsen, O., Boyden, E. S., \& Schultz, W. (2016). Dopamine Neuron-Specific Optogenetic Stimulation in Rhesus Macaques. Cell, 166(6), 1564-1571. doi:10.1016/j.cell.2016.08.024

Takeuchi, T., Duszkiewicz, A. J., Sonneborn, A., Spooner, P. A., Yamasaki, M., Watanabe, M., ... Morris, R. G. M. (2016). Locus coeruleus and dopaminergic consolidation of everyday memory. Nature, 537(7620), 357-362. doi:10.1038/nature 19325

Tan, P., He, L., Han, G., \& Zhou, Y. (2017). Optogenetic Immunomodulation: Shedding Light on Antitumor Immunity. Trends in Biotechnology, 35(3), 215-226. doi:10.1016/j.tibtech.2016.09.002

Tønnesen, J. (2013). Optogenetic cell control in experimental models of neurological disorders. Behavioural Brain Research, 255, 35-43. doi:10.1016/j. 
bbr.2013.07.007

Wingerchuk, D. M., Carter, J. L., Noseworthy, J. H., Lucchinetti, C., Rodriguez, M., Weinshenker, B. G., ... Derfuss, T. (2014). Multiple sclerosis: current and emerging disease-modifying therapies and treatment strategies. Mayo Clinic Proceedings, 89(2), 225-40. doi:10.1016/j.mayocp.2013.11.002

Zemelman, B. V, Lee, G. A., Ng, M., \& Miesenbö Ck, G. (2002). Neurotechnique Selective Photostimulation of Genetically ChARGed Neurons transmitted optical signal that can be decoded and transduced into electrical activity by only a subset of all illuminated neurons. The \&quot; receiver \&quot; of the optical signal. Neuron, 33, 15-22.

Zhang, J., Kramer, E. G., Mahase, S., Dutta, D. J., Bonnamain, V., Argaw, A. T., \& John, G. R. (2011). Targeting oligodendrocyte protection and remyelination in multiple sclerosis. The Mount Sinai Journal of Medicine, 78(2), 244-57. doi: $10.1002 / \mathrm{msj} .20244$

Ziemssen, T., Derfuss, T., de Stefano, N., Giovannoni, G., Palavra, F., Tomic, D., ... Schippling, S. (2016). Optimizing treatment success in multiple sclerosis. Journal of Neurology, 263(6), 1053-65. doi:10.1007/s00415-015-7986-y 